\title{
Children's Discrete Proportional Reasoning Is Related to Inhibitory Control and Enhanced by Priming Continuous Representations
}

Roberto A. Abreu-Mendoza ${ }^{1}$, Linsah Coulanges ${ }^{1 *}$, Kendell Ali $^{2}$, Arthur B. Powell ${ }^{3}$, and Miriam

\author{
Rosenberg-Lee ${ }^{1}$ \\ ${ }^{1}$ Department of Psychology, Rutgers University - Newark \\ ${ }^{2}$ Graduate School of Education, Rutgers University \\ ${ }^{3}$ Department of Urban Education, Rutgers University - Newark
}

\begin{abstract}
Author Note
We would like to thank Principal Rose, Ms. Crockett and all the teachers and staff at [Blinded] who helped in this study. We also thank Blessing Eluwa, Milagros Grados, Jessica Amato, Dima Karim, Isabel Paucar, Kainaat Anwar, and Catalina Guzman for their assistance with data collection and Elisee Doh for stimuli generation. *Linsah Coulanges is now at the Department of Psychology, University of Pittsburgh, USA.
\end{abstract}




\begin{abstract}
Children can successfully compare continuous proportions as early as age 4 , yet struggle to compare discrete proportions least to age 10 , especially when the discrete information is misleading. This study examined whether inhibitory control contributes to individual differences in discrete proportional reasoning and whether reasoning could be enhanced by priming continuous information. Forty-nine second-graders completed two tasks. In the Hearts and Flowers (H\&F) task, a measure of inhibition, children pressed either on the corresponding or opposite side depending on the identity of the displayed figure. In the Spinners task, a measure of proportional reasoning, children chose the spinner with the proportionally larger red area, across continuous and two discrete formats. In the discrete adjacent format, the continuous stimuli were segmented into sections, which could be compatible with the proportional information or misleading; the discrete mixed format interspersed the colored sections from the discrete adjacent conditions. Finally, two priming groups were formed. Children who saw the continuous immediately before the discrete adjacent format formed the Continuous-priming group ( $n=26)$. Children who saw discrete mixed immediately before the discrete adjacent format formed the Discrete-priming group $(n=23)$. Our results showed that children who performed better in the H\&F task also had better performance on the discrete counting misleading trials. Furthermore, children in the Continuous-priming group outperformed children in the Discrete-priming group, specifically in contexts where discrete information was misleading. These results suggest that children's proportional reasoning may be improved by fostering continuous representations of discrete stimuli and by enhancing inhibitory control skills.
\end{abstract}

Keywords: Proportional reasoning, Inhibitory control, Mathematical development 


\section{Proportional Reasoning in Childhood}

Children can reason about non-symbolic ratios several years before they can work easily with the same quantities represented symbolically. Before starting elementary school, children can reliably indicate which of two figures has the proportionally larger target area (Hurst \& Cordes, 2016), and kindergarteners can identify objects of differing sizes that have the same ratio of colored areas (Boyer \& Levine, 2015; Boyer, Levine, \& Huttenlocher, 2008). These tasks are thought to required proportional reasoning, that is, "understanding the multiplicative relationships between rational quantities" (Boyer, Levine, \& Huttenlocher, 2008, p. 1478).

Despite these capacities, when proportions are segmented into discrete elements, children often ignore multiplicative relationships and instead tend to focus on the absolute number of pieces. This bias is especially problematic when discrete and proportional information are at odds with each other. Children's performance plummets when counting information contradicts proportional information (Jeong, Levine, \& Huttenlocher, 2007), and even adults resort to counting strategies in these cases (Plummer, DeWolf, Bassok, Gordon, \& Holyoak, 2017). Thus, a critical step in correctly accessing non-symbolic proportional information is inhibiting misleading discrete information, suggesting that individuals who are better at resolving interferences in general, may show enhanced proportional reasoning abilities. Yet, to date, there is no empirical evidence for the link between individual differences in proportional reasoning and the executive function capacity of inhibitory control. In this study, we examine the contributions of inhibitory control to proportional reasoning in second-grade children, who had yet to receive any formal fraction instruction, particularly in those cases where there is misleading discrete information.

\section{Contributions of Inhibitory Control to Proportional Reasoning}


Emerging evidence suggests that inhibitory control is involved in successful processing of symbolic ratios (Avgerinou \& Tolmie, 2019; Coulanges et al., 2020; Gomez, Jimenez, Bobadilla, Reyes, \& Dartnell, 2015). Avgerinou and Tolmie (2019) found that eight-to-ten year old children's inhibitory control skills were positively related to their ability to compare rational numbers, both fractions and decimals. Importantly, children's inhibitory control skills were specifically related to their performance on comparisons that had misleading information, for example, trials where the largest fraction had the smallest denominator. These results add to the growing evidence suggesting that inhibitory control plays a role in reducing the impact of misleading information in numerosity processing more generally (Gilmore et al., 2013; Norris \& Castronovo, 2016) and even in scientific reasoning when content contradicts learners prior knowledge (Bascandziev, Tardiff, Zaitchik, \& Carey, 2018; Brookman-Byrne, Mareschal, Tolmie, \& Dumontheil, 2018). Here, we aim to investigate, for the first time in children, whether inhibitory control is related to non-symbolic proportional reasoning, especially in contexts with misleading discrete information.

Distinguishing between contexts with and without misleading discrete information may account for the mixed patterns of results when studies examine relations between inhibitory control and fraction understanding in adults. Matthews, Lewis, and Hubbard (2016) found that performance on a non-symbolic proportional reasoning task significantly predicted symbolic fraction skills even after controlling for performance on a flanker task, their measure of inhibitory control. However, they combined continuous and discrete stimuli and did not consider the independent contributions of consistent and inconsistent trials in their predictors or outcomes. Nor did they examine the effects of segmenting the non-symbolic continuous proportions. 
The lack of consensus on how best to measure inhibitory control (Lee \& Lee, 2019) may also contribute to the mixed evidence for the contribution of inhibitory control to proportional reasoning. For instance, Gomez et al. (2015) found a positive relationship between performance on a numerical Stroop task and fraction comparison task, a measure of symbolic proportional reasoning. But, the authors report that children's mathematical performance mediated this relationship, suggesting that inhibitory control did not uniquely drive fraction comparison performance. However, the mediation effect could have been due in part by the fact that their measure of inhibitory control involved numerical magnitude understanding skills, which might overestimate the relationship between inhibition and mathematical skills. To avoid potential confounds between measures, in the current study we employed a domain-general inhibitory control measure, the Hearts and Flowers task (Davidson, Amso, Anderson, \& Diamond, 2006; Wright \& Diamond, 2014). This task not only shows a clear developmental trajectory from ages 4 to 26 (Davidson et al., 2006), but also has appropriate concurrent validity with a latent variable of inhibition that comprises performance on the color-word and animal Stroop tasks (Brocki \& Tillman, 2014).

\section{Priming Effects in Non-symbolic Proportional Reasoning}

These individual difference studies of symbolic proportional reasoning suggest that successful non-symbolic proportional reasoning of discrete stimuli may also require inhibitory control to overcome interfering counting information, yet they do not identify the appropriate representation for processing discrete stimuli. One possibility is suggested by studies reporting reduced interference from counting information when children are primed with non-symbolic continuous representations of proportions. For example, Boyer and Levine (2015) examined whether presenting proportions in a continuous format could prompt proportional reasoning in 
discrete format trials that were presented after the continuous ones. They found that ten-yearolds, but not six- or eight-year-olds, benefited from being prompted with continuous proportions. Similarly, Hurst and Cordes (2018) reported that when five-year-olds saw continuous proportional stimuli before discrete stimuli, they successfully compared trials with and without discrete misleading information. However, this benefit of priming was not observed in three- or seven-year-olds. These results suggest that working with continuous stimuli can prompt proportional reasoning in subsequent discrete stimuli; however, the evidence is mixed about what age children benefit from continuous priming. The discrepancies between these studies may stem from the difficulty of the tasks employed. In both studies, only age groups not at ceiling or floor (chance level) benefited from the continuous priming; suggesting it may only be beneficial when there is sufficient variation within an age group. In the current study, we employed a proportion comparison task that is simpler than the match to sample task employed by Boyer and Levine (2015) but used a more challenging set of stimuli than those used in Hurst and Cordes (2018). Thus, this task may better afford detection priming effects in seven- and eight-year old children.

\section{The Current Study}

The goals of the study are three-fold. First, we aimed to provide empirical evidence for the presumed relationship between inhibitory control and proportional reasoning in contexts where proportional and whole number counting information were at odds. Thus, we hypothesize that better inhibitory control skills, measured by the Hearts and Flowers (H\&F) task, will correlate with better proportional reasoning in misleading contexts, while such correlation will be absent in contexts where there is no discrete information (i.e. comparisons of continuous stimuli) and when discrete information is congruent with proportional information. Second, 
although not originally designed to test this hypothesis, we leveraged our counterbalanced study design to further examine whether proportional reasoning could be enhanced by priming continuous information. For this exploratory analysis, we hypothesize that seeing continuous information in a non-symbolic proportion comparison task would facilitate performance on discrete comparisons. Finally, we examined whether the facilitating effects of continuous information were related to, or independent of, individual differences in inhibitory control. If these two effects are independent, it would suggest that improving proportional reasoning in children could be achieved by either providing the right representation or by helping children to overcome misleading counting strategies. Alternatively, if the facilitating effects of continuous priming mitigate effects of poor inhibitory control, then we might expect an interaction between these factors.

\section{Methods}

\section{Participants}

Forty-nine second-graders between the ages of 6.9 to 8.1 years (mean age $=7.53$ years, $\mathrm{SD}=0.30 ; 28$ boys and 21 girls) from a Blinded public school participated in this study. Eightynine percent of students at this school were eligible for free or reduced-price lunch. Originally, the full sample consisted of 52 children; however, three children were excluded because their age suggested that they were likely to have failed a grade $(n=1)$, or because they did not have the minimum number of useable trials in the experimental tasks $(n=2)$. All children's parents gave written informed consent and children gave oral assent for their participation. All protocols were approved by the Blinded institutional review board.

This sample is part of a larger study that aims to evaluate the effects of an after-school enrichment program on children's proportional reasoning skills. As a subset of second-graders in 
after-school program formed the training group, we invited all students in that grade to participate in the current study, so the remainder would form the control group. From the total population of 57 second-graders at the school, 52 parents $(91 \%)$ provided consent. Sensitivity power analyses indicated that the final sample size $(n=49)$ enables detecting medium to large correlations (Pearson's $r>.38$ ), medium to large within-subject differences (Cohen's $d>.61$ ), and large between-subjects differences (Cohen's $d>.82$ ) using an alpha $=.05$ and power $=.80$.

\section{Materials}

Standardized Mathematics Assessment. To evaluate children's mathematical achievement, we used the Addition and Subtraction Math Fluency subtests from the Wechsler Individual Achievement Test - Third Edition (WIAT-III, Wechsler, 2009). In each test, children answered as many addition (subtraction) problems as they could in one minute. The dependent variable for these subtests was the Math Fluency composite score, which combined the gradenormed scores of each subtest.

Proportional Reasoning. We implemented a computerized version of the "Spinners Task" (Jeong et al., 2007) to measure children's proportional reasoning. In this task, children indicated which of two spinners had the proportionally larger red area. The task started with onscreen instructions, which the experimenter read aloud to the child. Then, the experimenter presented the child a set of three stickers and told them they could win more stickers during the task. Next, the experimenter explained that they would see two spinners, one on the left and one on the right, which would spin, and they had to select one. After spinning, if the arrow pointed to the red part of the spinner, they would win a sticker, but would lose one if it stopped on the blue part. Children were instructed that their task was to pick the spinner they wanted to play with to win another sticker. Next, children saw a video of two spinners of equal size spinning and 
coming to stop with the arrow pointed to the red part of the spinner with the proportionally larger red area, while the arrow pointed to the blue part of the other spinner, which had proportionally smaller red area. Children were reminded that they would have won a sticker if they had chosen the spinner where the arrow landed on the red part but would have lost one if they had chosen the other spinner, which landed on blue. Before the experimental trials began, children performed a practice trial to familiarize themselves with pressing the corresponding key for each side. In the experimental trials, the spinners did not spin; however, at the end of each of the three blocks, there was an additional trial in which the spinners spun, and the arrow always landed in the red portion on the side the child selected, regardless of proportion presented. These trials were not included in the analyses, and all children received six stickers at the end of the task, as they had always made the "correct" choice on the three sticker trials. After completing a sticker trial, children were reminded of the instructions before starting the next block.

The 12 proportions used by Jeong et al. (2007) were presented in three different format blocks for a total of 36 experimental trials. In the Continuous format, each spinner had only two continuous sections, one red and one blue (Figure 1A). In the Discrete adjacent format, the two continuous parts were broken in discrete, but adjacent sections of red and blue segments (Figure 1B). In the Discrete mixed format, the red and blue segments were interspersed. (Figure 1C). In the discrete blocks, the number of segments was manipulated so that in half the trials, the spinner with the larger number of red pieces was also the one with the proportionally larger red area (Counting consistent trials), while in the other half, the spinner with the fewer red pieces was the one with the proportionally larger red area (Counting misleading trials). Although "counting" information could not be meaningfully assessed in the Continuous format, trials that had the same proportions as the Counting consistent trials of the Discrete formats were considered 
Continuous "Counting consistent" trials by convention; similarly, Continuous trials that show the same proportions as the Counting misleading trials were considered Continuous "Counting misleading" trials. Figures 1A \&1B illustrates the correspondence between Continuous and Discrete "Counting consistent" (i.e. $2 / 7$ vs. 1/5, left panels) and "Counting misleading" (i.e. 3/5 vs. $4 / 8$, right panels) stimuli.

For all formats, we also manipulated the size of the individual spinners to prevent children from relying on the absolute size of the red area, to make their selections. Thus, on half of the trials of each format, the physically larger spinner also had the proportionally larger red area (Size congruent trials), while on the other half, the opposite pattern held, that is, the smaller spinner was the one with the proportionally larger red area (Size incongruent trials). Spinners could be 6, 9, or $12 \mathrm{~cm}$ in diameter. For Size congruent trials, the proportionally larger spinner was always the $12 \mathrm{~cm}$ spinner and the other spinner could be 6 or $9 \mathrm{~cm}$, while for the Size incongruent trials, the proportionally larger spinner was the $6 \mathrm{~cm}$ and the other spinner was 9 or $12 \mathrm{~cm}$. Proportions pairs (Size congruent or incongruent) were counterbalanced across participants.

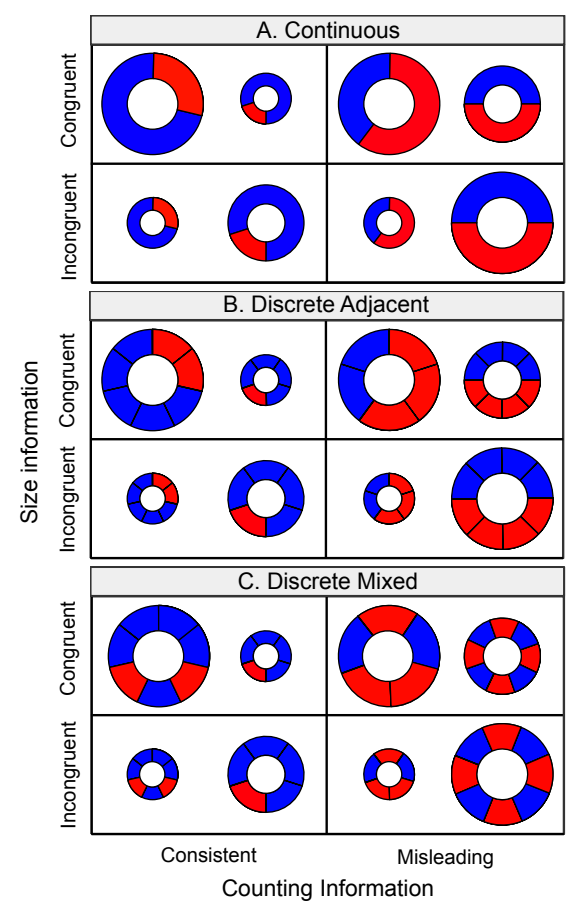


Figure 1. Examples of the stimuli used in the Spinners task, where participants had to choose the spinner with the proportionally larger red area. All spinner pairs on the left column show a $2 / 7$ vs. $1 / 5$ comparison, while pairs on the right column show a $3 / 5$ vs. $4 / 8$ one. For each of the three formats, the upper row presents pairs where the larger spinner is the one with the proportionally larger red area (Size congruent trials); the lower row shows pairs where the opposite pattern holds (Size incongruent trials). Finally, for the two discrete formats, columns also represent the counting information: On the left column, there are pairs of spinners where the spinner with the larger number of pieces is the correct answer (Counting consistent trials, 2/7 vs. $1 / 5)$. On the right column, there are pairs where the spinner with the larger number of pieces is the incorrect one (Counting misleading trials, $3 / 5$ vs. 4/8).

For all participants, the Continuous condition was presented first, while the presentation order of the two discrete blocks was counterbalanced across participants, generating the priming conditions. Size congruency and counting consistency were counterbalanced within each block; however, due to an experimental error, the four condition combinations (i- Size congruentCounting consistent, ii- Size congruent-Counting misleading, iii- Size incongruent-Counting consistent, and iv- Size incongruent-Counting misleading) were unevenly distributed within each block. Across participants, some blocks had 3 trials in each condition (3-3-3-3), while others were 2-4-4-2 or 4-2-2-4, respectively. For further details, see Supplementary Analyses Table S1, where we provide summary details of the three different trial distribution groups (hereafter Design-group factor), which we use to assess any effects of this experimental error. Importantly, none of the reported effects are due to this error.

For all conditions, trials started with a blank screen presented for $500 \mathrm{~ms}$, followed by the pair of spinners. Spinners remained on the screen until the child responded by pressing one of two possible keys, ' $z$ ' for the left spinner and ' $m$ ' for the right one. Within each block, half of the correct responses were presented on the left and the other half were presented on the right. 
The dependent variable for this task was the proportion of correct responses (accuracy). Following Wright \& Diamond, (2014), and for consistency with the inhibitory control measure, when computing accuracy we exclude anticipatory (reaction times, RT, shorter than $250 \mathrm{~ms}$ ) and outlier responses (RT 3 SD above individual's mean). After applying these criteria, one participant from the full sample did not have at least one trial from each type and was excluded from the final sample. Thus, among the forty-nine children of the final sample, we analyzed 1713 $(97.11 \%)$ out of a total of 1764 trials.

Priming Manipulation. Participants were randomly assigned to one of two groups that showed the formats of the Spinners task in different orders. Children who saw the Discrete adjacent block immediately after the Continuous format, formed the Continuous-priming group $(\mathrm{n}=26)$. This order might promote proportional reasoning in the Discrete adjacent trials.

Children who saw the Continuous format, but then the Discrete mixed, before ending with the Discrete adjacent formed the Discrete-priming group $(n=23)$. This order might promote the use of counting strategies.

Inhibitory Control Measure. Children's inhibitory control was assessed with the Hearts \& Flowers (H\&F) task (Brocki \& Tillman, 2014; Davidson et al., 2006; Wright \& Diamond, 2014). This computerized task consisted of three blocks, presented in the following fixed order: Congruent, Incongruent, and Mixed. The experimenter read aloud the onscreen instructions to the children. In the Congruent block, they were instructed to press the key on the same side as where the target (Hearts) appeared, using the same keys as in the Spinners task: the letter ' $\mathrm{z}$ ' for the left side and ' $m$ ' for the right side. In the Incongruent block, children were instructed to press the key on the opposite side as where the target (Flowers) appeared. In the Mixed block, children saw interspersed hearts and flowers and were asked to respond according to the previously 
learned rules. At the beginning of the Congruent and Incongruent blocks, there were two example trials. First, the corresponding figure (heart or flower) appeared on the right, then, on the left. The target images remained on the screen until the child pressed the correct key. The first two blocks comprised 12 trials each, which randomly presented the corresponding figure on each side six times. The third, Mixed, block comprised 33 trials, and the first trial of this block was always a heart presented at the right side. Subsequent trials randomly presented each figure 16 times, eight times on each side. We considered this last block as the measure of inhibitory control as prior research finds that performance in the Mixed block is strongly correlated with a latent variable of inhibition $(r=.71)$, while performance in the Congruent and Incongruent blocks are negative $(r=-.03)$ or weakly associated $(r=.17)$, respectively (Brocki \& Tillman, 2014).

Before the experimental blocks, there were four practice trials for the Congruent and Incongruent blocks, while there were eight for the Mixed block. All trials started with a fixation cross presented for $500 \mathrm{~ms}$, followed by a blank screen for $500 \mathrm{~ms}$. Then, within a horizontal rectangle presented at the center of the screen, children saw the target image (a heart or flower, depending on the block). After $750 \mathrm{~ms}$, the rectangle and the image disappeared and were replaced by a blank screen that lasted $500 \mathrm{~ms}$ before the next trial began. Children had to respond during this $1250 \mathrm{~ms}$ period.

Following the same procedure as in the Spinners task, the dependent variable was the proportion of correct responses (accuracy), excluding anticipatory and outlier responses. One participant of the full sample lost more $20 \%$ of the trials and was excluded from the final sample. Thus, we analyzed $2742(98.17 \%)$ out of a total of 2793 trials from the forty-nine participants. 
Positive Attitude Toward Math (PAM) questionnaire. As part of the larger training study, we also collected the PAM questionnaire to evaluate the relationship between children's attitudes toward math and learning gains. Following Chen et al. (2018), we used the five level Likert-type but employed emojis to help connote the response options. The measure comprises 12 questions: the first six evaluate children's attitude toward math, while the other six evaluate their general attitude toward academics.

\section{Statistical analyses}

Statistical analyses were conducted in R 3.5.3 (R Core Team, 2019). As measures of effect sizes, we reported the absolute values of Cohen's $d$ for $t$-tests and generalized eta squared $\left(\eta^{2}\right)$ for ANOVAs and ANCOVAs. For analyses of variance, generalized eta squared is preferred over partial eta squared, as it allows to compare between and within-subjects designs (Bakeman, 2005). For this measure of effect size, values of .020 are considered small, .130 as medium, and .260 as large.

\section{General Procedure}

Children performed the tasks in the following fixed order: Addition subtest, Subtraction subtest, the Spinners task, the H\&F task, and the Positive Attitude toward Math (PAM) questionnaire (Chen et al., 2018), which is not reported here. Both, the Spinners and the H\&F tasks were presented using PsychoPy2 Experiment Builder v1.90.3 (Pierce, 2007). Children were evaluated individually by trained experimenters in quiet corners of a large room at the children's school (maximum four children at a time). The evaluation lasted approximately 25 min.

\section{Results}

\section{Mathematical Performance}


Children's mean WIAT Math Fluency composite score, which combines the Addition and Subtraction subtests, was $80.65(\mathrm{SD}=9.96$, range $=63-106)$, indicating that children performed below average relative to their grade.

\section{Proportional Reasoning}

To evaluate how children's performance was affected by the spinners' format, size and counting information, we performed a repeated measures ANOVA with Format (Continuous, Discrete adjacent, and Discrete mixed), Size (congruent and incongruent), and Counting (consistent and misleading) as within-subjects factors and accuracy as the dependent variable. This analysis yielded main effects of Size $\left(F(1,48)=31.95, p<.001, \eta^{2}=.066\right)$ and Counting $\left(F(1,48)=35.54, p<.001, \eta^{2}=.096\right)$, which were qualified by an interaction between these factors $\left(F(1,48)=5.58, p=.022, \eta^{2}=.005\right.$, Figure 2A). Specifically, children's performance was particularly low in trials where the smaller spinner, with fewer pieces, had the proportionally larger red area. Children's performance in these trials was not better than chance $(t(48)=0.93, p$ $=.354$, Cohen's $d=0.13)$. Although there was no main effect of Format $(F(2,96)=0.05, p=$ $\left..955, \eta_{\mathrm{g}}^{2}<.001\right)$, as performance across conditions was similar when collapsing both types of Counting trials (consistent and misleading), there was an interaction between Format and Counting $\left(F(2,96)=14.79, p<.001, \eta_{\mathrm{g}}^{2}=.049\right.$, Figure $\left.2 \mathrm{~B}\right)$. Counting strategies are only possible when the sections of the spinners are broken into segments, thus, this effect should be absent in the Continuous format but present in the discrete ones. Consistently, children's performance was impaired by in the misleading conditions of the discrete formats, but not in the Continuous condition. 
A.

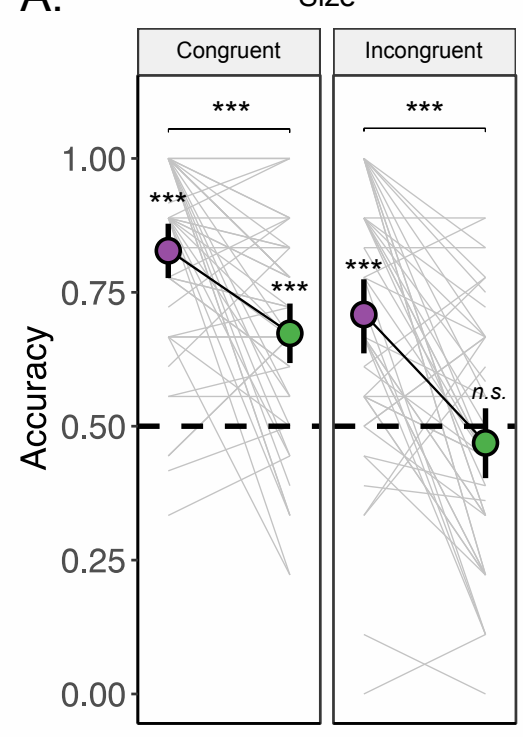

B.

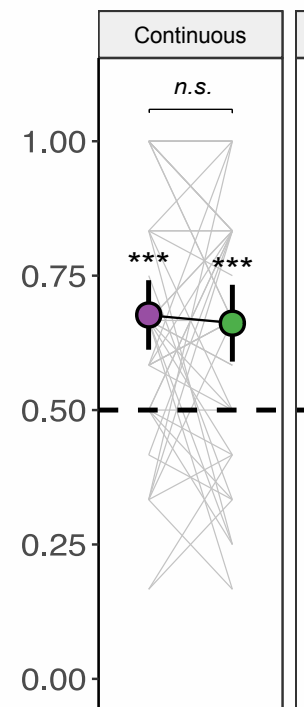

Format

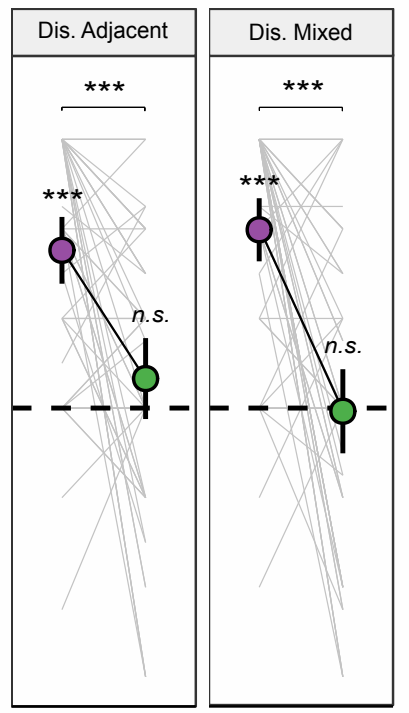

Counting Information

Consistent Misleading

Figure 2. Performance on the Spinners task. A. The effect of counting information differed between the two size conditions. Children's performance was particularly low in trials where the smaller spinner with fewer pieces had the proportionally larger red area (Size incongruent and Counting misleading trials); in these trails, children's performance was no better than chance. In contrast, performance was highest in trials where the larger spinner with the larger number of pieces had the proportionally larger red area (Size congruent and Counting consistent trials). B. Counting information affected children's performance differently across formats. In the Continuous format, as there was no counting information, there was no difference between the two types of counting trials. In contrast, for the Discrete (dis.) adjacent and Discrete mixed formats, misleading counting information impaired performance and was no better than chance. Note. ${ }^{* * *} p<.001$

\section{Inhibitory Control}

Next, to examine the effect of cognitive control demands on children's performance in the H\&F task, we performed a repeated measures ANOVA with Block (Congruent, Incongruent, and Mixed) as a within-subjects factor and accuracy as the dependent variable. This analysis yielded a main effect of $\operatorname{Block}\left(F(2,96)=46.1, p<.001, \eta_{\mathrm{g}}^{2}=.335\right)$. Children had a higher accuracy on the Congruent than Incongruent block, which was, in turn higher than the Mixed 
block. In fact, performance on the Mixed block was not better than chance $(t(48)=1.64, p=.11$, Cohen's $d=0.23$, Figure 3 ).

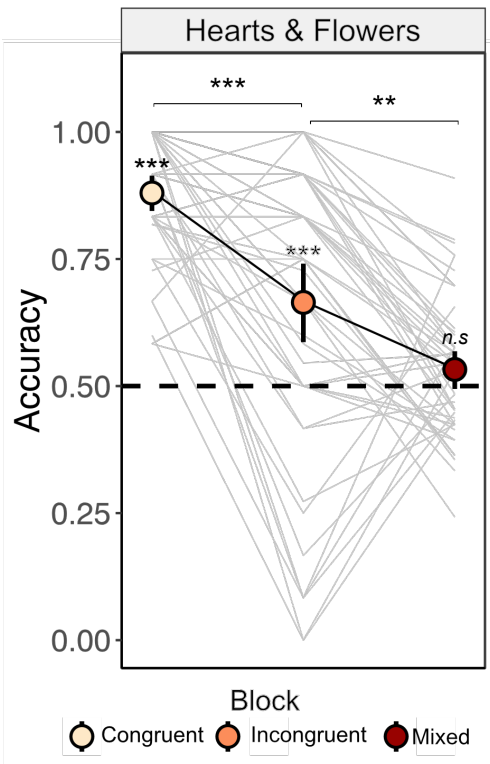

Figure 3. Performance on the Hearts and Flowers task. Children's performance varied depending on the inhibitory control demands of each block. Their performance was the highest in the Congruent block, in which they had to press a key on the same side as where the figures appeared, followed by the Incongruent block, where they had to press a key on the opposite side. Children's performance was the lowest in the Mixed block, where they saw interspersed Congruent and Incongruent trials. Note. ${ }^{* * *} p<.001,{ }^{* *} p<.01$

\section{Relations Between Proportional Reasoning and Inhibitory Control}

To investigate whether better inhibitory control results in better proportional reasoning skills, we examined Pearson correlations between the mixed trials of the H\&F task and performance in the Counting misleading trials of the Discrete adjacent format. In our sample, children on average were around chance level (50\%) in the Mixed block, and only one of them was at an extreme level (>90\%). By contrast, in the Incongruent block, there were 15 children above $90 \%$ and another five below $10 \%$, suggesting that in this condition, many children are not engaging inhibition but rather are just applying a single response rule. These results further support using the Mixed block as the measure of children's inhibitory control. For the Spinners 
tasks, we were particularly interested in the Counting misleading trials of the Discrete adjacent format as the only difference between the Continuous trials and these trials is the segmentation lines, allowing a more direct exploration of the effect of discrete information on proportional reasoning. Furthermore, as opposed to the Counting misleading trials of the Discrete mixed format, these trials do not require a mental manipulation to bring the red pieces together.

As expected, children who performed better on these taxing comparisons also had higher accuracy on mixed trials $(r(47)=.32, p=.023$, Figure 4 upper panel). Furthermore, if this correlation is driven by the contribution of inhibitory control to avoiding inappropriate counting strategies, inhibitory control should not be associated with comparison performance in contexts where these strategies are not feasible (Continuous trials) or where using them leads to the correct answer (Discrete adjacent, Counting consistent). Accordingly, the Pearson correlation between performance in the Mixed block of H\&F and the 'misleading' trials of the Continuous format was not significant $(r(47)=.13, p=.374$, Figure 4 left lower panel), nor was it significantly related to performance on the consistent trials of the Discrete adjacent format $(r(47)$ $=.16, p=.262$, Figure 4 right lower panel). However, the Steiger's Z-test (Steiger, 1980) for non- independent group values indicated that the two latter correlation coefficients were not different from the one between performance in the Mixed block and Counting misleading Discrete adjacent trials $(p s>.30)$. These results were not driven by the distribution of trials seen (see Supplementary Analyses Table S1). 


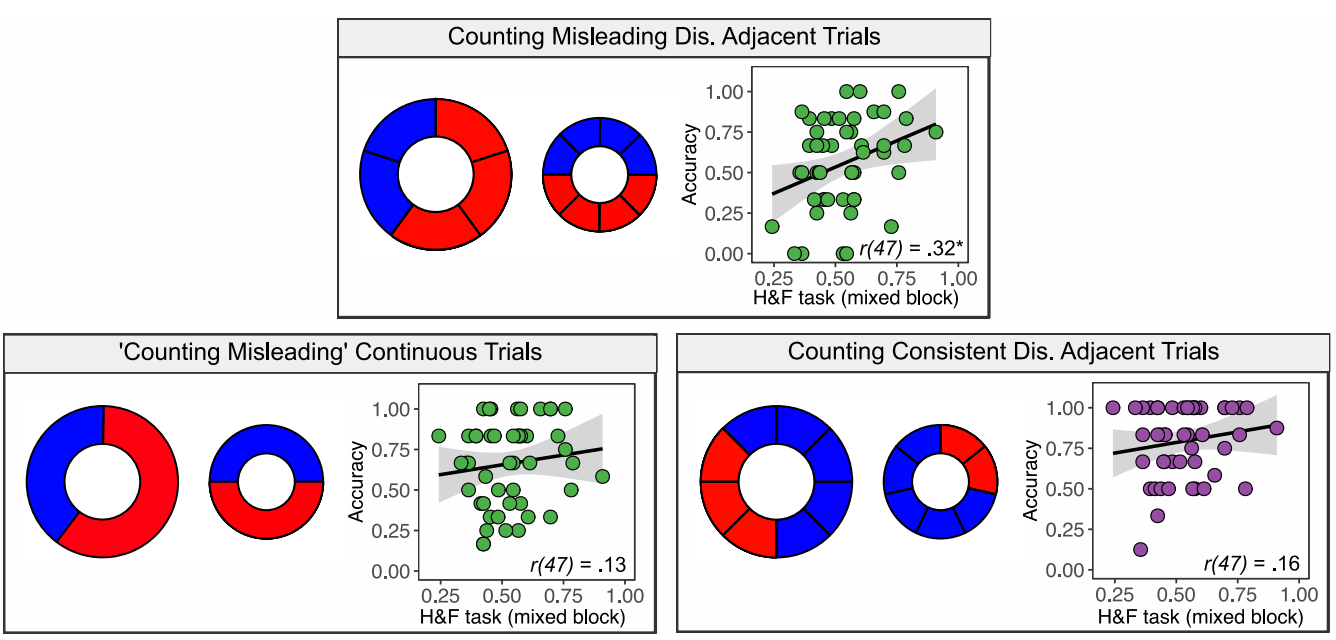

Figure 4. Associations between proportional reasoning and inhibitory control. Children with greater inhibitory control skills, measured by the Mixed block of the Hearts and Flowers (H\&F) task, also performed better on the Counting misleading Discrete (dis.) adjacent trials. In contrast, there was no significant correlation with same ratios in Continuous Counting misleading nor with Counting consistent Discrete trials Note. ${ }^{*} p<.05$

\section{Exploratory Analyses}

Effects of Continuous and Discrete Priming. The two priming groups did not differ in age $(t(47)=1.32, p=.19$, Cohen's $d=0.38)$, math performance $t(47)=0.98, p=.33$, Cohen's $d$ $=0.28)$, or inhibitory control skills $((t(47)=.13, p=.90$, Cohen's $d=0.04)$, see Table 1 .

Table 1

Groups' mean age and control measures

\begin{tabular}{lcccc}
\hline & $\begin{array}{c}\text { Continuous-priming } \\
\text { group }(\mathrm{n}=26) \\
M(S D)\end{array}$ & $\begin{array}{c}\text { Discrete-priming } \\
\text { group }(\mathrm{n}=23)\end{array}$ & & \\
& $7.48(0.31)$ & $7.60(0.27)$ & 1.32 & .19 \\
\hline Age (years) & $79.34(11.23)$ & $82.13(8.30)$ & 0.98 & .33 \\
WIAT Math Fluency & $0.53(0.12)$ & $0.53(0.16)$ & 0.13 & .90 \\
H\&F mixed trials &
\end{tabular}

Note. WIAT $=$ Wechsler Individual Achievement Test, H\&F $=$ Hearts \& Flowers.

To explore whether comparing continuous proportions had a short-term facilitating effect on children's discrete proportional reasoning, we added Priming-group as a between-subject factor to the original repeated measures ANOVA, which considered the effects of format, size 
and counting on Spinners task accuracy. In addition to the same main effects and interactions of the previous ANOVA, this mixed-design repeated-measures ANOVA yielded a significant interaction between Priming-group and Counting $\left(F(1,47)=4.90, p=.032, \eta^{2}=.014\right)$, which was qualified by a marginal three-way interaction between Priming-group, Counting, and Format $\left(F(2,94)=2.73, p=.071, \eta^{2}=.009\right)$. Importantly, there was no significant interaction between Priming-group and Format $\left(F(2,94)=2.23, p=.11, \eta_{\mathrm{g}}^{2}=.008\right)$, suggesting that performance in each format was not affected by presentation order. Next, we performed separate follow-up ANOVAs for each of the formats with Priming-group as a between-subject factor and Counting consistency as a within-subject factor. The ANOVA for the Discrete adjacent format showed no main effect of Priming-group $\left(F(1,47)=0.84, p=.36, \eta^{2}=.001\right)$, indicating that even though children in the Continuous-priming group saw the Discrete adjacent trials as the second block, while children in the Discrete-priming saw it as the third block, differences between their performance are likely not due to fatigue leading to overall lower performance later in the experiment. Instead, we found the expected main effect of Counting $(F(1,47)=27.83, p<.001$, $\left.\eta_{\mathrm{g}}^{2}=.20\right)$, but it was qualified by an interaction between Priming-group and Counting $(F(1,47)$ $=11.24, p=.002, \eta_{\mathrm{g}}^{2}=.094$, Figure 5A). Specifically, children in the Continuous-priming group performed significantly better on the Discrete misleading trials $(t(47)=2.67, p=.010$, Cohen's $d$ $=0.76)$, but marginally worse in the Discrete consistent trials $(t(47)=1.67, p=.101$, Cohen's $d$ $=0.48$ ) relative to children in the Discrete-priming group. Furthermore, in contrast to the Discrete group, children in the Continuous group performed significantly above chance in the misleading trials $(65 \%, t(25)=3.46, p=.002$, Cohen's $d=0.68)$. Taken all together, these results indicate that priming continuous representations may allow children to extend their continuous strategies to discrete comparisons, especially for misleading stimuli. Finally, the imbalanced 
number of trials did not drive these effects: Children who saw the three different trial

distributions (Design-group factor) were evenly distributed across the two priming groups $\left(X^{2}(2\right.$, $N=49)=0.40, p=.820$, Table S2), and there was no main effect of Design-group or significant interactions (all p’s > .12, Table S3).

The ANOVAs for the other two formats only showed the expected effects: for the Discrete mixed, only a main effect of Counting $\left(F(1,47)=35.48, p<.001, \eta_{\mathrm{g}}^{2}=.298\right)$; for Continuous, no main effects or interactions. Further, there were no main effects or interactions with Priming-group (all $p \mathrm{~s}>.20$ ) for either format. Coupled with the lack of difference in age, math ability, and inhibitory control capacity between the priming groups, these results suggest that facilitation effects of proportional priming are not driven by confounding individual differences between the groups.
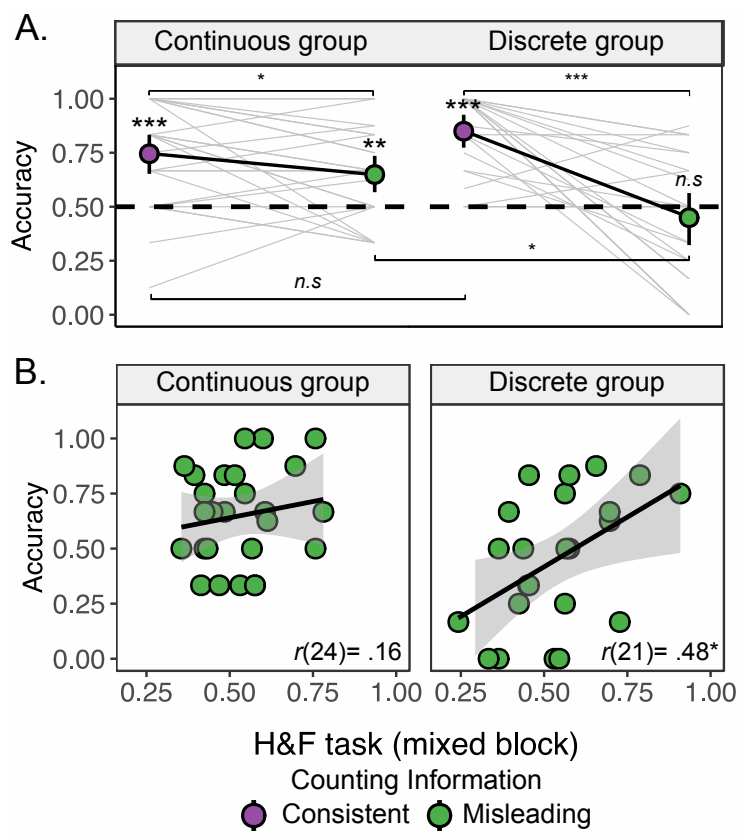

Figure 5. Priming groups' performance in the Discrete adjacent trials. A. For the Discrete misleading trials, children in the Continuous-priming group performed above chance and significantly better than their peers in the Discrete-priming group. B. Inhibitory control, as measured by Hearts \& Flowers (H\&F) task, was associated with performance in the Counting misleading trials for children in the Discrete-priming group, but not for the Continuous-priming group. Note. ${ }^{* * *} p<.001,{ }^{* *} p<.01,{ }^{*} p<.05$ 
Relations Between Priming and Inhibitory Control. Finally, to examine whether the facilitation effect of continuous information was related or independent of individual differences in inhibitory control, we performed Pearson correlations between performance in the Counting misleading trials of the Discrete adjacent format and performance in the mixed trials of the H\&F task, for each group separately. These analyses revealed a significant correlation for the Discretepriming group $(r(21)=.48, p=.022$, Figure 5B left panel $)$, but not in the Continuous-priming group $(r(24)=.16, p=.428$, Figure 5B right panel). However, an ANCOVA with Group as a between-subjects factor, performance on the Mixed block of the H\&F tasks as a co-variate, and performance in the Counting misleading trials of the Discrete adjacent format as the dependent variable, showed the expected main effect of $\operatorname{Group}\left(F(1,45)=8.30, p=.006, \eta_{\mathrm{g}}^{2}=.156\right)$, and $\mathrm{H} \& \mathrm{~F}$ as a significant covariate $\left(F(1,45)=6.42, p=.015, \eta^{2} \mathrm{~g}=.129\right)$, but no interaction between Group and performance in the $\operatorname{H\& F}\left(F(1,45)=1.35, p=.252, \eta^{2}=.029\right)$, suggesting that both priming and inhibitory control demonstrate independent contributions to proportional reasoning.

\section{Discussion}

In this study, we investigated the relationship between inhibitory control and proportional reasoning in contexts where whole-number and proportional information contradict each other. Although research has shown that inhibitory control is related to incongruent information in other numerical and mathematical domains (Gilmore et al., 2013; Gilmore, Keeble, Richardson, \& Cragg, 2015), to the best of our knowledge, our results are the first to demonstrate that children who had greater inhibitory control skills also had better proportional reasoning skills specifically when required to overcome misleading discrete information. As part of our exploratory analyses, we also examined whether continuous information could prime proportional processing and consequently enhance second-graders' discrete proportional 
reasoning. Consistent with previous studies (Boyer \& Levine, 2015; Hurst \& Cordes, 2018), we found that children who saw the Continuous format immediately before the Discrete adjacent outperformed children who saw the Discrete mixed immediately before the Discrete adjacent, specifically in Discrete misleading trials.

\section{Inhibitory Control Supports Overcoming Misleading Discrete Information}

Which abilities help children suppress the interfering effects of misleading discrete information on proportional reasoning? A recent theoretical framework proposes that inhibitory control helps leaners avoid using intuitive incorrect responses and instead promote analyzing problems more carefully (Van Dooren \& Inglis, 2015). Growing evidence supports this framework: inhibitory control contributes to adolescents' reasoning about counterintuitive scientific facts (Brookman-Byrne et al., 2018), children's performance in numerical tasks with misleading visual information (Gilmore et al., 2013), and children's avoidance of incorrect strategies in measurements tasks (Ren, Lin, \& Gunderson, 2019). In the context of discrete proportional reasoning, one possible intuitive response strategy is counting the segments. At least in adulthood, eye-tracking studies have shown that when proportions are presented in a discrete format, they elicit counting strategies (Plummer et al., 2017). Since performance on the H\&F task shows a moderate relationship with the Discrete adjacent Counting misleading trials of the Spinners task that might elicit a counting strategy, we suggest that the role of inhibitory control in proportional reasoning is to help children avoid using this strategy and instead, focus on the proportional information. Consistently, children's inhibitory control was weakly and not significantly associated with proportional reasoning when there was no misleading information (Continuous trials) and when both discrete and proportional information were consistent (Discrete adjacent consistent trials). However, differences between correlation coefficients did 
not reach significance, suggesting that inhibitory control may also contribute to these other trial types. Future studies, with larger samples, are needed to provide definitive evidence of either a general role for inhibition in proportional reasoning or a specific role in misleading discrete cases.

This framework also helps to reconcile our results with previous studies where inhibitory control did not play a role in symbolic proportional reasoning (Gomez et al., 2015; Matthews et al., 2016). For example, Matthews et al. (2016) reported that the relation between non-symbolic proportional reasoning and fraction comparison skills was independent of inhibitory control. However, their measure of proportional reasoning combined continuous and discrete representations, and it did not distinguish between those trials in which the proportional and discrete information were consistent from those in which they were not, potentially diluting the role of inhibitory control. Furthermore, in studies of fractions and decimals that do distinguish these types of trials do find correlation with measures of inhibitory control (Avgerinou \& Tolmie, 2019; Coulanges et al., 2020; Gomez et al., 2015), the authors used a measure of inhibitory control that required symbolic number comparison, the numerical Stroop task, confounding the contribution of inhibitory control with numerical skills. Recent studies suggest that numerical and non-numerical inhibition tasks have differential contributions in other math domains (e.g., whole number arithmetic, Gilmore et al., 2015). Future studies should collect both types of measures in order to elucidate whether these two forms of inhibition have independent or overlapping contributions to proportional reasoning.

\section{Continuous Priming Bolsters Discrete Proportional Reasoning}

As opposed to discrete representations, continuous representations of proportions do not elicit counting strategies (Plummer et al., 2017). Thus, studies have investigated whether these 
representations could prime proportional reasoning skills when children make judgments of discrete proportions (Boyer \& Levine, 2015; Hurst \& Cordes, 2018), finding that continuous representations are successful in improving children's performance. However, the evidence is mixed about the age in which children are sensitive to this priming. While one study found it at age five but not at age three or seven (Hurst \& Cordes, 2018), another study only found it at age ten but not at age six or eight (Boyer \& Levine, 2015). One reason for this discrepancy might be the different difficulty levels of the tasks employed. While there was a ceiling effect in the oldest group's performance of the first study, there was a floor effect in the younger groups' performance of the second study.

Here, we found that when there is enough room for improvement, seven-year-olds' discrete proportional reasoning benefits from continuous priming. Notably, the two groups were matched on age, math performance, and inhibitory control, and performed comparably on the Continuous and Discrete mixed formats. Yet, seeing the continuous information was sufficient to improve performance above chance for discrete misleading problems among seven-year-olds, a level of performance not seen even among ten-year-olds (Jeong et al., 2007). Further, the priming effect was independent of the contributions of inhibitory control, suggesting that children's proportional reasoning could be improved by either fostering the appropriate strategies through continuous representations or by inhibiting the inappropriate ones. Importantly, as our study was not initially designed to examine the effects of priming, presentation order of the three formats was confounded with the type of priming that children received. Nevertheless, the specificity of our results for the misleading trials in the Discrete adjacent block, rather than poorer performance for all trials in the block, argues against a fatigue interpretation of these results. Still, future studies of priming effects should present the block of interest in the same position 
across groups, as done previously (Hurst \& Cordes, 2018), and include larger samples. And conversely, studies primarily focused on continuous versus discrete non-symbolic formats must be cognizant of these order effects.

\section{Experimental and Educational Considerations}

Although the Spinners task has been widely used to measure children's proportional reasoning (Hurst \& Cordes, 2018; Jeong et al., 2007), one alternative strategy to solve this task is to compare the angle formed by the red sections of the spinners, rather than the proportions. The spinners with the proportionally larger red area are also those with larger angles. However, based on how the size of the spinners and counting information impaired children's performance, it is unlikely that children were using this strategy. Future eye-tracking studies could provide a deeper insight on the kind of strategies that children use while comparing non-symbolic proportions.

Another concern is that in contrast to previous reports, children of this study came from a disadvantaged socioeconomic background and had lower math and inhibitory control skills (Davidson et al., 2006). Timed measures of addition and subtraction fluency were particularly low $($ mean $=80.65)$, below the one standard deviation cutoff $($ standard score $=85)$ used in some studies to identify students with mathematical disabilities (Iuculano et al., 2015; Swanson, 2012). However, their socioeconomic background and the time of the year when evaluated likely account for their low math scores. We assessed children during the first weeks of their secondgrade year. Prior work on the academic losses over the summer, a phenomenon called 'summer dip,' shows that these losses are especially pronounced in children with lower SES, relative to their peers with higher SES (Alexander, Entwisle, \& Olson, 2004). Interestingly, despite these limitations, they showed comparable performance to that of the group of eight-year-olds in 
Jeong's study (2007). This pattern of results suggest that proportional reasoning may be less affected by socioeconomics factors, making it a promising target for educational interventions. Future research should investigate proportional reasoning in samples that include the complete spectrum of math ability and could consider more comprehensive assessments of math knowledge. Given the role of multiplicative understanding in proportional reasoning (Kouba, 1989), assessing multiplication and division might be more informative, than addition and subtraction, especially in older children with more familiarity with these arithmetic operations.

Our results also speak to an ongoing debate about how best to teach fractions, suggesting advantages for continuous over discrete representations. Instruction programs that introduce fractions with area models (e.g., segmented pie charts) have been criticized as they mislead children to think of fractions as parts of a whole and prompt them to use counting strategies (Obersteiner, Dresler, Bieck, \& Moeller, 2019). As an alternative to the part-whole approach, a measurement perspective has been proposed (Davydov \& Tsvetkovich, 1991; Powell, 2019; Wong \& Evans, 2008). From this perspective, fractions serve as a more accurate measurement of a reference, specifically in those cases when the multiplicative relation between the reference and the measuring unit does not result in a whole number. In one practical example of this perspective (Powell, 2019), children start learning about proportions with physical, continuous representations (e.g., Cuisenaire rods). Using these rods, children observe the multiplicative relation between a reference and the measuring units; for instance, they can observe that the length of one black rod equals the length of three red rods plus one white rod, which is half of the size of the red rod. As a result, they can observe that one black rod equals seven halves of a red rod. After being familiarized with the rods, children start working with symbolic representations of proportions. 
Further support for the importance of continuous representations in educational contexts comes from correlational studies reporting that better acuity in placing fraction magnitudes on number is related to better understanding of fractions (Siegler, Thompson, \& Schneider, 2011). Finally, there is also evidence from brief-intervention studies (Hamdan \& Gunderson, 2017), showing that children who go through a 30-min training on how to represent fractions in a number line improve their fraction comparison skills, while children who use area models do not. Our results suggest that these approaches may be effective because they facilitate proportional representation of symbolic fractions. Future studies should examine this contention using more intensive interventions based on the measurement perspective.

\section{Conclusions}

Our study provides the first evidence from individual difference designs for the relationship between inhibitory control and discrete proportional reasoning. Moreover, our results add to the growing body of evidence demonstrating the short-term, facilitating effect of continuous proportions on discrete proportional reasoning, extending for the first time to sevenyear-olds. Finally, we find that facilitating effects of continuous priming and individual differences in inhibitory control are both independent contributors to discrete proportional reasoning. 


\section{References}

Alexander, K. L., Entwisle, D. R., \& Olson, L. S. (2004). Schools, achievement, and inequality: A seasonal perspective. Summer Learning: Research, Policies, and Programs, 23(2), 2551. https://doi.org/10.4324/9781410610362

Avgerinou, V. A., \& Tolmie, A. (2019). Inhibition and cognitive load in fractions and decimals. British Journal of Educational Psychology, 1-17. https://doi.org/10.1111/bjep.12321

Bakeman, R. (2005). Recommended Effect Size Statistic. Behavior Research Methods, 37(3), 379-384. https://doi.org/10.3758/BF03192707

Bascandziev, I., Tardiff, N., Zaitchik, D., \& Carey, S. (2018). The role of domain-general cognitive resources in children's construction of a vitalist theory of biology. Cognitive Psychology, 104(March), 1-28. https://doi.org/10.1016/j.cogpsych.2018.03.002

Boyer, T. W., \& Levine, S. C. (2015). Prompting children to reason proportionally: Processing discrete units as continuous amounts. Developmental Psychology, 51(5), 615-620. https://doi.org/10.1037/a0039010

Boyer, T. W., Levine, S. C., \& Huttenlocher, J. (2008). Development of Proportional Reasoning: Where Young Children Go Wrong. Developmental Psychology, 44(5), 1478-1490. https://doi.org/10.1037/a0013110

Brocki, K. C., \& Tillman, C. (2014). Mental Set Shifting in Childhood : The Role of Working Memory and Inhibitory Control. Infant and Child Development, 604(June), 588-604. https://doi.org/10.1002/icd

Brookman-Byrne, A., Mareschal, D., Tolmie, A. K., \& Dumontheil, I. (2018). Inhibitory control and counterintuitive science and maths reasoning in adolescence. PLoS ONE, 13(6), 1-19. https://doi.org/10.1371/journal.pone.0198973 
Chen, L., Bae, S. R., Battista, C., Qin, S., Chen, T., Evans, T. M., \& Menon, V. (2018). Positive Attitude Toward Math Supports Early Academic Success: Behavioral Evidence and Neurocognitive Mechanisms. Psychological Science, 29(3), 390-402.

https://doi.org/10.1177/0956797617735528

Coulanges, L., Abreu-Mendoza, R. A., Varma, S., Uncapher, M., Gazzaley, A., Anguera, J., \& Rosenberg-Lee, M. (2020). Linking inhibitory control to math achievement via comparison of conflicting decimal numbers. Retrieved June 5, 2020, from psyarxiv.com/qsuz9

Davidson, M. C., Amso, D., Anderson, L. C., \& Diamond, A. (2006). Development of cognitive control and executive functions from 4 to 13 years: Evidence from manipulations of memory, inhibition, and task switching. Neuropsychologia, 44, 2037-2078.

Davydov, V. V., \& Tsvetkovich, Z. H. (1991). The object sources of the concept of fractions. In V. V. Davydov \& L. P. Steffe (Eds.), Psychological Abilities of Primary School Children in Learning Mathematics. Soviet Studies in Mathematics Education. Volume 6. (pp. 86-147). Reston, VA: National Council of Teachers of Mathematics.

Gilmore, C., Attridge, N., Clayton, S., Cragg, L., Johnson, S., Marlow, N., ... Inglis, M. (2013). Individual Differences in Inhibitory Control, Not Non-Verbal Number Acuity, Correlate with Mathematics Achievement. PloS ONE, 8(6), e67374.

Gilmore, C., Keeble, S., Richardson, S., \& Cragg, L. (2015). The role of cognitive inhibition in different components of arithmetic. ZDM - Mathematics Education, 47(5), 771-782. https://doi.org/10.1007/s11858-014-0659-y

Gomez, D. M., Jimenez, A., Bobadilla, R., Reyes, C., \& Dartnell, P. (2015). The effect of inhibitory control on general mathematics achievement and fraction comparison in middle school children. ZDM - International Journal on Mathematics Education, 47, 801-811. 
Hamdan, N., \& Gunderson, E. A. (2017). The number line is a critical spatial-numerical representation: Evidence from a fraction intervention. Developmental Psychology, 53(3), 587-596. https://doi.org/10.1037/dev0000252

Hurst, M. A., \& Cordes, S. (2016). Rational-number comparison across notation: Fractions, decimals, and Whole Numbers. Journal of Experimental Psychology: Human Perception and Performance, 42(2), 281-293. https://doi.org/10.1037/xhp0000140

Hurst, M. A., \& Cordes, S. (2018). Attending to Relations : Proportional Reasoning in 3- to. Developmental Psychology, 54(3), 428-439.

Iuculano, T., Rosenberg-Lee, M., Richardson, J., Tenison, C., Fuchs, L., Supekar, K., \& Menon, V. (2015). Cognitive tutoring induces widespread neuroplasticity and remidates brain function in children with mathematical learning disabilities. Nature Communications, 6(8453). https://doi.org/10.1038/ncomms9453

Jeong, Y., Levine, S. C., \& Huttenlocher, J. (2007). The Development of Proportional Reasoning: Effect of Continuous Versus Discrete Quantities. Journal of Cognition and Development, 8(2), 237-256.

Kouba, V. L. (1989). Children's solution strategies for equivalent set multiplication and division word problems. 147-158.

Lee, K., \& Lee, H. W. (2019). Inhibition and Mathematical Performance: Poorly Correlated, Poorly Measured, or Poorly Matched? Child Development Perspectives, 13(1), 28-33. https://doi.org/10.1111/cdep.12304

Matthews, P. G., Lewis, M. R., \& Hubbard, E. M. (2016). Individual Differences in Nonsymbolic Ratio Processing Predict Symbolic Math Performance. Psychological Science, 27(2), 191-202. https://doi.org/10.1177/0956797615617799 
Norris, J. E., \& Castronovo, J. (2016). Dot display affects approximate number system acuity and relationships with mathematical achievement and inhibitory control. PLoS ONE, 11(5), 1-15. https://doi.org/10.1371/journal.pone.0155543

Obersteiner, A., Dresler, T., Bieck, S. M., \& Moeller, K. (2019). Understanding Fractions: Integrating Results from Mathematics Education, Cognitive Psychology, and Neuroscience. In A. Norton \& M. W. Alibali (Eds.), Constructing number: merging perspectives from psychology and mathematics education. (pp. 135-162). https://doi.org/10.1007/978-3-03000491-0_7

Pierce, J. W. (2007). PsychoPy- Psychophysics software in Python. Journal of Neuroscience Methods, 162, 8-13.

Plummer, P., DeWolf, M., Bassok, M., Gordon, P. C., \& Holyoak, K. J. (2017). Reasoning strategies with rational numbers revealed by eye tracking. Attention, Perception, and Psychophysics, 79(5), 1426-1437. https://doi.org/10.3758/s13414-017-1312-y

Powell, A. B. (2019). How does a fraction get its name? Revista Brasileira de Educação Em Ciências e Educação Matemática, 3(3), 700-713.

R Core Team. (2019). R: A language and environment for statistical computing, reference index version 3.5.3.

Ren, K., Lin, Y., \& Gunderson, E. A. (2019). The role of inhibitory control in strategy change: The case of linear measurement. Developmental Psychology, 55(7), 1389-1399. https://doi.org/10.1037/dev0000739

Siegler, R. S., Thompson, C. A., \& Schneider, M. (2011). An integrated theory of whole number and fractions development. Cognitive Psychology, 62(4), 273-296. https://doi.org/10.1016/j.cogpsych.2011.03.001 
Steiger, J. H. (1980). Tests for comparing elements of a correlation matrix. Psychological Bulletin, 87, 245-251. https://doi.org/10.1037//0033-2909.87.2.245

Swanson, H. L. (2012). Cognitive profile of adolescents with math disabilities: Are the profiles different from those with reading disabilities? Child Neuropsychology, 18(2), 125-143. https://doi.org/10.1080/09297049.2011.589377

Van Dooren, W., \& Inglis, M. (2015). Inhibitory control in mathematical thinking, learning and problem solving: a survey. ZDM - Mathematics Education, 47(5), 713-721. https://doi.org/10.1007/s11858-015-0715-2

Wechsler, D. (2009). Wechsler Individual Achievement Test-Third Edition. San Antonio, TX: Pearson.

Wong, M., \& Evans, D. (2008). Fractions as a measure. In M. Goos, R. Brown, \& K. Makar (Eds.), Navigating Currents and Charting Directions. Proceedings of the 31st Annual Conference of the Mathematics Education Research Group of Australasia (pp. 597-603).

Wright, A., \& Diamond, A. (2014). An effect of inhibitory load in children while keeping working memory load constant. Frontiers in Psychology, 5(March), 1-9. https://doi.org/10.3389/fpsyg.2014.00213 